\title{
Kyliniella latvica Skuja (Stylonemataceae, Stylonematophyceae), un rodófito indicador de buena calidad del agua
}

\author{
María Eugenia García-Fernández ${ }^{1, *}$, Iara Seguí-Chapuis ${ }^{2}$ y Marina Aboal ${ }^{1}$ \\ ${ }^{1}$ Laboratorio de Algología. Departamento de Biología Vegetal. Facultad de Biología. Universidad de Murcia. \\ E-30100 Murcia. España. \\ ${ }^{2}$ Departamento de Botánica. Facultad De Ciencias. Campus de Fuentenueva. Universidad De Granada. 18071 \\ Granada. España. \\ * Corresponding author: marujegf@gmail.com
}

Received: 29/12/11

Accepted: 2/3/12

\begin{abstract}
The Rhodophyta Kyliniella latvica Skuja (Stylonemataceae, Stylonematophyceae), a good water quality indicator Kyliniella latvica Skuja is a wide spread Rhodophyta scarcely reported because of its seasonality. Its presence is always linked to low-nutrient environments, and may be considered a good indicator of oligotrophy. The species has been collected in lakes and streams in America and Europe. This is the first record of the filamentous mature thallus for Spain.
\end{abstract}

Key words: Stylonematales, Rhodophyta, Kyliniella, seasonality, distribution, SE Spain.

\section{RESUMEN}

Kyliniella latvica Skuja (Stylonemataceae, Stylonematophyceae), un rodófito indicador de buena calidad del agua

Kyliniella latvica Skuja es un rodófito de amplia distribución pero escasamente citado debido a su carácter estacional. Su presencia siempre está ligada a ambientes bajos en nutrientes, y podría considerarse un buen indicador de oligotrofía. La especie ha sido recolectada en lagos y arroyos de America y Europa. Esta es la primera vez que se cita para España de la parte madura filamentosa.

Palabras clave: Stylonematales, Rhodophyta, Kyliniella, estacionalidad, distribución, SE España.

\section{INTRODUCCIÓN}

Las algas rojas continentales (Rhodophyta) han sido escasamente estudiadas en la Península Ibérica hasta tiempos recientes. Aparte de los trabajos de Margalef (recogidos por Álvarez-Cobelas, 1984; Ballesteros et al., 1985) se han publicado escasas aportaciones sobre el tema, que incluyen el estudio de áreas más o menos extensas como la cuenca del río Segura (Aboal, 1989a), o zonas del NE y SE de España (Sabater et al.,1989), junto con nuevas citas para la flora: Batrachospermum boryanum Sirodot (Prefasi \& Aboal, 1994), Batrachospermum atrum (Hudson) Harvey (Aboal et al., 1995), Hildenbrandia angolensis Welwitsch ex West \& G. S. West (Ros et al., 1997), Thorea violacea Bory de Saint-Vincent (Egidos \& Aboal, 2003) o Batrachospermum arcuatum Kylin (Marco \& Aboal, 2008).

El género Kyliniella Skuja es monoespecífico y pertenece al orden Stylonematales que incluye además los géneros Stylonema Reinsch, Ban- 
giopsis F. Schmitz in Engler \& Prantl, Chroodactylon Hansgirg, Chroothece Hansgirg in Wittrock \& Nordstedt, Purpureofilum J. A. West, Zuccarello \& J. L. Scott in J. A. West, Zuccarello, J. L. Scott, J. Pickett-Heaps \& G. H. Kim, Rhodosorus Geitler, Rhodospora Geitler, y Rufusia D. E. Wujek \& P. Timpano (Sheath \& Wehr, 2003). En este orden se incluyen los rodófitos unicelulares, filamentosos o pseudofilamentosos, con varios cloroplastos, con o sin pirenoide y reproducción por división celular o monósporas (Yoon et al., 2006). Anteriormente el género Kyliniella se incluía dentro del orden Porphyriadiales junto con los géneros Cyanidium, Flintiella, Porphyridium, Chroodactylon, Chroothece y Rufusia (Sheath \& Wehr, 2003), pero los análisis moleculares actuales han esclarecido la filogenia de los mayores linajes de las algas rojas continentales y modificado su clasificación.

Kyliniella se caracteriza principalmente por presentar una parte basal discoidal, de donde parten filamentos uniseriados no ramificados que pueden alcanzar varios centímetros, con células que pueden elongarse lateralmente en forma de rizoides, que contienen cloroplastos parietales acintados de color rosáceo (Bourrelly, 1970).

Ha sido citada escasamente en cursos de agua de localidades de Europa y América (Tabla 1), aunque se le atribuye una distribución cosmopolita (Bourrelly, 1970). En este trabajo se aporta una nueva cita de esta especie para la flora algal continental española.

Tabla 1. Comparación de poblaciones de Kyliniella latvica del río Alhárabe con la bibliografía. Comparison of the populations of Kyliniella latvica in Alhárabe river with the literature.

\begin{tabular}{|c|c|c|c|c|c|c|c|c|c|}
\hline \multicolumn{2}{|r|}{ Poblaciones } & $\begin{array}{c}\varnothing \text { Célula } \\
(\mu \mathrm{m})\end{array}$ & $\begin{array}{l}\text { Long. Célula } \\
\qquad(\mu \mathrm{m})\end{array}$ & $\begin{array}{l}\varnothing \text { Rizoide } \\
(\mu \mathrm{m})\end{array}$ & $\begin{array}{l}\text { Long Rizoide } \\
\qquad(\mu \mathrm{m})\end{array}$ & $\begin{array}{c}\varnothing \text { Filamento } \\
(\mu \mathrm{m})\end{array}$ & A mbiente & $\begin{array}{c}\text { Características } \\
\text { ecológicas }\end{array}$ & Referencias \\
\hline \multirow{3}{*}{$\frac{8}{8}$} & $\begin{array}{c}\text { New } \\
\text { Hampshire } \\
\text { (Estados } \\
\text { Unidos) }\end{array}$ & 7.4-14.8 & 4.9-11.1 & 7.4-9.9 & $17.3-24.7$ & $12.4-32.1$ & A rroyo & \multirow{2}{*}{$\begin{array}{l}\text { Poco común en la } \\
\text { zona litoral de los } \\
\text { ríos, en la región } \\
\text { de bosque } \\
\text { caducifolio de } \\
\text { Norte A mérica }\end{array}$} & $\begin{array}{l}\text { Flint } 1953, \mathrm{~V} \text { is \& } \\
\text { Sheath } 1993\end{array}$ \\
\hline & $\begin{array}{c}\text { Rhode Island } \\
\text { (Estados } \\
\text { Unidos) }\end{array}$ & $8.0-11.0$ & $5.0-9.0$ & $7.0-10.0$ & $18.0-25.0$ & 24.0 & A rroyo & & $\begin{array}{l}\text { Sheath \& } \\
\text { B urkholder } 1985\end{array}$ \\
\hline & $\begin{array}{l}\text { Río San } \\
\text { Francisco, } \\
\text { Canastra } \\
\text { (B rasil) }\end{array}$ & - & - & - & - & - & A rroyo & $\begin{array}{l}\text { Recolectada sólo } \\
\text { en invierno en } \\
\text { zonas con } \\
\text { sustratos poco } \\
\text { rocosos y con } \\
\text { poca abundancia } \\
\text { de especies }\end{array}$ & $\begin{array}{l}\text { Necchi et al. } \\
2003\end{array}$ \\
\hline \multirow{5}{*}{$\begin{array}{l}\frac{8}{0} \\
\frac{0}{3} \\
\text { ய }\end{array}$} & $\begin{array}{l}\text { Lago U sma } \\
\text { (Letonia) }\end{array}$ & $9.0-10.0$ & $14.0-22.0$ & 7.4-9.9 & $10.0-150.0$ & $10.0-19.1$ & Laguna & $\begin{array}{l}\text { Epífita sobre } \\
\text { Chara, } \\
\text { Phragmites y } \\
\text { Scirpus }\end{array}$ & Skuja 1926 \\
\hline & $\begin{array}{c}\text { Río } \\
\text { Alhárabe, } \\
\text { Murcia } \\
\text { (España) }\end{array}$ & $8.3-29.9$ & $17.4-40.66$ & $8.9-12.5$ & $156.3-170$ & $35.78-50.92$ & A rroyo & $\begin{array}{l}\text { Filamentos } \\
\text { primero fijados a } \\
\text { las rocas en } \\
\text { zonas de } \\
\text { corriente y } \\
\text { después flotan en } \\
\text { aguas } \\
\text { remansadas } \\
\text { oligotróficas }\end{array}$ & Este trabajo \\
\hline & A ustria & - & - & - & - & - & Laguna & A guas alcalinas & $\begin{array}{l}\text { Geitler 1954, } \\
\text { B ourrelly } 1970\end{array}$ \\
\hline & Francia & - & - & - & - & - & Laguna & - & B ourrelly 1970 \\
\hline & Suecia & - & - & - & - & - & Laguna & - & Israelson 1938 \\
\hline
\end{tabular}




\section{MATERIALES Y MÉTODOS}

Kyliniella latvica fue recolectada en el río Alhárabe situado en la localidad de Moratalla (NO Murcia) en las coordenadas UTM WH 9130 (Fig. 1). Este río nace a $1440 \mathrm{~m}$ de altura y se incorpora al río Segura $50 \mathrm{~km}$ más abajo. Se caracteriza por tener un curso breve, un caudal irregular, superficie de $348875 \mathrm{~km}^{2}$ y una pendiente media del $24 \%$ (López-Bermúdez, 1973). Los materiales litológicos predominantes de la cuenca son calizas, dolomías y margas. La vegetación de ribera está dominada por saucedas y helófitos como Arundo donax L. y Phragmites australis (Cav.) Trin. ex Steud. En zonas de aguas remansadas o en las represas proliferan los céspedes monospecíficos de Zannichellia palustris L. o Potamogeton coloratus L. (Aboal, 1989b). El clima es mediterráneo subhúmedo con menos de $550 \mathrm{~mm}$ de precipitación anual, con picos en otoño y en primavera. La temperatura media anual es de $11.7^{\circ} \mathrm{C}$.
Dentro del contexto de un proyecto extensivo realizado a lo largo de un año para caracterizar los principales factores ambientales que afectan al desarrollo de las comunidades de algas en el río Alhárabe, se recolectaron muestras que se conservaron en frío hasta la llegada al laboratorio. En el campo se midieron algunas variables limnológicas (temperatura, $\mathrm{pH}$, conductividad, velocidad de la corriente y profundidad). Paralelamente se tomaron muestras de agua, se filtraron con filtros de $0.45 \mu \mathrm{m}$ de tamaño de poro y se analizó su composición química (fósforo total, ortofosfato, $\mathrm{NO}_{3}^{-}, \mathrm{NO}_{2}^{-}$y Si) con los kits de análisis de aguas Merck (Spectroquant).

En el laboratorio se realizó el estudio morfológico y morfométrico del material vegetal con un microscopio óptico dotado de contraste interdiferencial y cámara digital (OLYMPUS BX50). Parte del material se conservó en seco y se depositó en el Herbario MUB-ALGAS. El estudio morfométrico (realizado en 40 células) se basó en los siguientes parámetros: diámetro y longitud

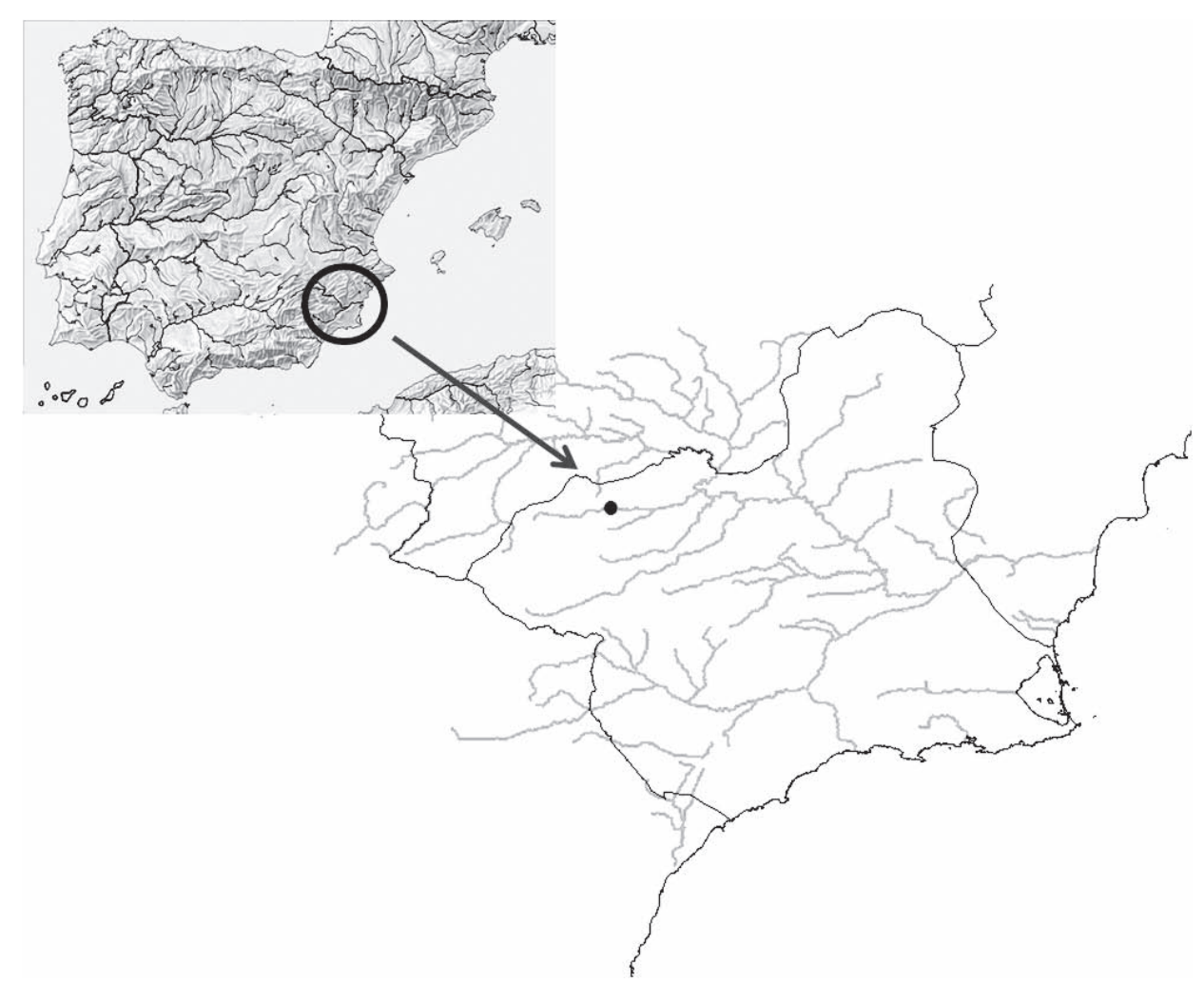

Figura 1. Localización del río Alhárabe (Moratalla, Murcia). Location of the Alhárabe river (Moratalla, Murcia). 
celular, diámetro y longitud del rizoide y diámetro del filamento. Además, se valoró la actividad fosfatásica microscópicamente (la producción de las enzimas fosfatasas es una estrategia eficiente para la mineralización del fósforo orgánico disponible en el medio por las algas) con BCIPT/ TNBT (5-bromo-4-cloro-3-indoxil fosfato y cloruro de nitro-azul de tetrazolio) (Elwood \& Whitton, 2007). Como resultado de la tinción para la actividad fosfomonoesterasa, el BCIPT/TNBT se hidrolizó y se obtuvo un precipitado azuladopurpúreo y por tanto la localización de la enzima. Kyliniella fue incubada en $4 \mathrm{ml}$ de solución BCIPT/TNBT a temperatura ambiente $\left(20^{\circ} \mathrm{C}\right)$ durante $15-20$ minutos antes de añadir $0.5 \mathrm{M} \mathrm{NaOH}$ y lavar con agua desionizada. Las muestras se observaron al microscopio.

\section{RESULTADOS}

Kyliniella latvica se desarrolla durante un corto periodo de tiempo a finales del invierno o inicios de la primavera. El pH del agua es básico y la conductividad moderada. La temperatura puede alcanzar valores de hasta $9.4{ }^{\circ} \mathrm{C}$ en verano, pero en invierno, aunque no llega a congelarse, puede descender hasta $4.7^{\circ} \mathrm{C}$ (Aboal et al., 2005). El caudal es el parámetro que más oscila durante el periodo invierno-primavera. Las concentra- ciones de nutrientes son escasas, los silicatos y los nitratos son los que aparecen en concentraciones mayores mientras que el ortofosfato y nitritos permanecen en niveles menores (Tabla 2). La especie posee una elevada actividad fosfatasa (BCIPT/TNBT) a lo largo de todo el año que le permite convertir el fósforo orgánico en inorgánico para poder incorporarlo.

Las matitas son de un color pardoamarillento y pueden alcanzar varios centímetros de longitud. A pesar de que aparentemente suelen vivir fijadas a plantas, en el río Alhárabe son más frecuentes sobre rocas y pueden flotar libremente en su madurez y acumularse en los remansos. La parte basal, adherida al substrato, es discoidal y de ella parte un filamento no ramificado de 35.78-50.92 $\mu \mathrm{m}$ de diámetro o con ramas cortas (Tabla 1). Las células son más o menos discoidales de 8.3-29.9 $\mu \mathrm{m}$ de diámetro, 17.4-40.66 $\mu \mathrm{m}$ de altura, con varios cloroplastos acintados parietales, de color rosado. Poseen una gruesa vaina mucilaginosa en la que abundan bacterias epífitas. Es frecuente observar la formación de rizoides a partir de las células del filamento (Fig. 2). Las dimensiones del material recolectado en el río Alhárabe son más grandes que las publicadas para las muestras de los ríos americanos (Flint, 1953; Vis \& Sheath, 1993; Sheath \& Burkholder, 1985; Necchi et al., 2003) y se asemejan a las de la localidad tipo de Letonia (Skuja, 1926).

Tabla 2. Características físico-químicas del agua del río Alhárabe durante el periodo invierno-primavera. Physical and chemical characteristics of Alhárabe river water during the winter-spring period.

\begin{tabular}{lccc}
\hline Características & Media & Mínimo & Máximo \\
físico-químicas & & & 9.70 \\
\hline $\mathrm{T}^{\mathrm{a}}\left({ }^{\circ} \mathrm{C}\right)$ & 7.03 & 8.20 & 8.40 \\
$\mathrm{pH}$ & 8.35 & 515 & 785 \\
$\mathrm{Conductividad}(\mu \mathrm{S} / \mathrm{cm})$ & 649 & 3.90 & 64.50 \\
Caudal $(\mathrm{L} / \mathrm{s})$ & 39.48 & 3.90 & 8.40 \\
Profundidad $(\mathrm{cm})$ & 6.95 & 14.26 & 63.69 \\
Radiación $(\mathrm{PAR})\left(\mu \mathrm{E} / \mathrm{m}^{2} / \mathrm{s}\right)$ & 38.9 & 3.39 & 4.40 \\
Alcalinidad $(\mathrm{meq} / \mathrm{L})$ & 4.02 & 2.85 & 2.86 \\
$\mathrm{NO}-(\mathrm{mg} / \mathrm{L})$ & 2.85 & 6.03 & 10.99 \\
$\mathrm{NO}-(\mu \mathrm{g} / \mathrm{L})$ & 8.5 & 2.42 & 3.19 \\
$\mathrm{Si}(\mathrm{mg} / \mathrm{L})$ & 2.8 & 41.03 & 71.23 \\
Ortofosfato $(\mu \mathrm{g} / \mathrm{L})$ & 56.13 & 55.46 & 92.22 \\
Fósforo total $(\mu \mathrm{g} \mathrm{P} / \mathrm{L})$ & 73.84 & & \\
\hline
\end{tabular}




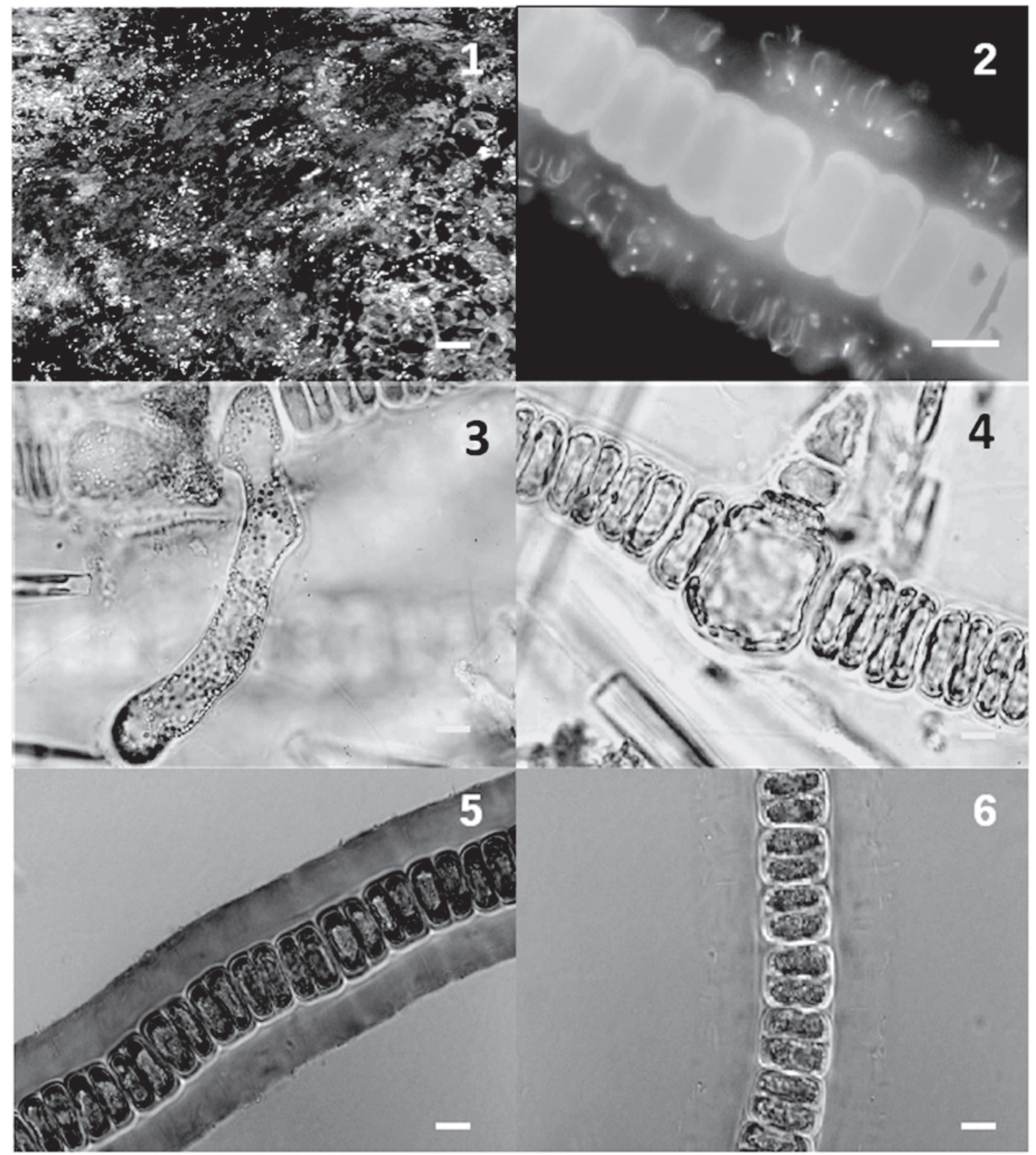

Figura 2. 1. Aspecto general de las poblaciones epilíticas de Kyliniella latvica en el río Alhárabe (escala $5 \mathrm{~cm}$ ). 2. Bacterias epífitas en la vaina mucilaginosa con microscopía de fluorescencia (escala $10 \mu \mathrm{m}$ ). 3. Rizoide lateral (escala $10 \mu \mathrm{m})$. 4 . Detalle de la formación de una pequeña rama (escala $10 \mu \mathrm{m}$ ). 5. Coloración negruzca que indica la transformación de fósforo orgánico en inorgánico por las enzimas fosfatasa alcalinas (BCIPT/TNBT) (escala $10 \mu \mathrm{m}$ ). 6. Control de la tinción BCIPT/TNBT, no se aprecia coloración (escala $10 \mu \mathrm{m})$. 1. General view of the epilithic populations of Kyliniella latvica in Alhárabe river (scale $5 \mathrm{~cm}$ ). 2. Epiphytic bacteria in the mucilaginous sheath with fluorescence microscopy (scale $10 \mu \mathrm{m}$ ). 3. Lateral rhizoid (scale $10 \mu \mathrm{m}$ ). Small branch formation (scale $10 \mu \mathrm{m}$ ). 5. Black staining due to the transformation of organic to inorganic phosphorus by alkaline phosphatases (BCIPT/TNBT) (scale $10 \mu \mathrm{m}$ ). 6. BCIPT/TNBT staining control, no colour (scale $10 \mu \mathrm{m}$ ). 


\section{DISCUSIÓN}

Kyliniella latvica ha sido citada en cursos de agua de Rhode Island y New Hampshire en Estados Unidos (Flint, 1953; Sheath \& Burkholder, 1985; Vis \& Sheath, 1993), en ríos de Brasil (río San Francisco) (Necchi et al., 2003), en el lago Usma de Letonia (Skuja, 1926), y en lagos de Suecia (Israelson, 1938), Austria y Francia (Geitler, 1954; Bourrelly, 1970). En España Cambra cita la presencia de los estados juveniles sobre Cladium mariscus L. en el lago de Basturs (Cambra, 1989; Cambra, 1991). Aunque Bourrelly (1970) le atribuye una distribución cosmopolita sería conveniente realizar un estudio de la especie en profundidad para tratar de dilucidar si se trata de una especie con gran variabilidad morfológica y ecológica o podría tratarse de varios taxones próximos. La distribución de la especie es probablemente mucho más amplia en nuestro país pero su marcada estacionalidad y su corto periodo de desarrollo probablemente dificulten su identificación.

Las especies acompañantes más frecuentes de Kyliniella son Chaetophora incrassata (Hudosn) Hazen, que tiene un desarrollo estacional, y $R i$ vularia biassolettiana Meneghini ex Bornet \& Flahault que puede observarse todo el año. La sencillez de su identificación y su presencia ligada a la escasez de nutrientes de las cabeceras de ríos calcáreos, parecen aconsejar incluirla en la lista de especies indicadoras de condiciones oligotróficas.

\section{AGRADECIMIENTOS}

Este trabajo ha sido financiado por la Comunidad Autónoma de Murcia (PEPLAN 2007-2011) y por la Fundación Séneca de la Región de Murcia (0572/PI/07).

\section{REFERENCIAS}

ABOAL, M. 1989a. Aportación al conocimiento de las algas epicontinentales del SE de España. II. Rodofíceas (Rhodophyceae). Lazaroa, 11: 115-122.

ABOAL, M. 1989b. Flora algal del río Benamor (cuenca del Segura, SE de España). Limnetica, 5: 1-11.
ABOAL, M., E. LÓPEZ-JIMÉNEZ \& A.D. ASENCIO. 1995. Batrachospermum atrum (Huds.) Harv. (Batrachospermales, Rodophyceae), novedad para la flora algal epicontinental española. Anales del Jardín Botánico de Madrid, 53(1): 121-123.

ABOAL, M., M. A. PUIG \& A. D. ASENCIO. 2005. Production of microcystins in calcareous Mediterranean streams: The Alhárabe, Segura river Basin in S.E. Spain. Journal of Applied Phycology, 17(3): 231-243.

ÁLVAREZ-COBELAS, M. 1984. Catálogo de las algas continentales españolas II: Craspedophyceae, Cryptophyceae, Chrysophyceae, Dinophyceae, Euglenophyceae, Haptophyceae, Phaeophyceae, Rhodophyceae, Xanthophyceae. Acta Botanica Malacitana, 9: 27-40.

BALLESTEROS, E., C. F. BOUDOURESQUE, M. P. BOUDOURESQUE, M. BRUGUÉS, J. CATALÁN, F. A. COMÍN, J. CAMBRA, R. M. CROS, M. COMELLES, M. DELGADO, M. HERNÁNDEZ, X. LLIMONA, J. MOLERO, M. A. RIBERA, J. ROMERO, X. TOMÁS \& F. TORRELLA.1985. Història natural dels Països Catalans: Plantes inferiors, vol. 4. Enciclopèdia Catalana S. A. Barcelona. 558 pp.

BOURRELLY, P. 1970. Les Algues d'eau douce. Les algues bleues et rouges. III. Ed. Boubée. París. 512 pp.

CAMBRA, J. 1989. Estudi sobre les algues epifitiques en sistemes lacustres. Ph.D. Thesis. University of Barcelona. 546 pp.

CAMBRA, J. 1991. Contribució a l'estudi de les algues epifítiques dels estanys de Banyoles, Basturs i Estanya. Orsis, 6: 27-44.

EGIDOS, A. I. \& M. ABOAL. 2003. Thorea violacea (Thoreaceae, Rhodophyceae) en fuentes del Marjal Pego-Oliva, Comunidad Valenciana. Nueva cita para la flora española. Anales del Jardín Botánico de. Madrid, 60: 27-32.

ELWOOD, N. \& B. WHITTON. 2007. Importance of organic phosphate hydrolyzed in stalks of the lotic diatom Didymosphenia gemianta and the possible impact of atmospheric and climatic changes. $\mathrm{Hy}$ drobiologia, 592: 121-133.

FLINT, L.H. 1953. Kyliniella in America. Phytomorphology, 3: 76-80.

GEITLER, L. 1954. Die Süsswasserbangiaceae Kyliniella latvica und ihr obligater bakterieller Bewohner. Österreichisches Botanisches Zeitschrift., 101: 304-314. 
ISRAELSON, G. 1938. Kyliniella latvica, in Schweden gefunden nebst neuen Fundorten für ein paar im Süsswasser auftrettende Bangioideen. Svensk Botanisk Tidskrift, 5: 20-23.

LÓPEZ BERMÚDEZ, F. J. 1973. La vega alta del Segura. Clima, hidrología y geomorfología. Publicaciones del Dpto. De Geografía. Univ. Murcia. 288 pp.

MARCO, S. \& M. ABOAL. 2008. Batrachospermum arcuatum (Batrachospermaceae, Floridophyceae) en el Parque Natural del Marjal de Pego-Oliva. Nueva cita para la flora algal de las aguas continentales españolas. ALGAS. Boletín de la Sociedad Española de Ficología, 39: 35-37.

NECCHI-JÜNIOR, O., L. H. Z. BRANCO \& C. C. Z. BRANCO. 2003. Ecological distribution of stream macroalgal communities from a drainage basin in the serra da Canastra Nacional Park, Minas Gerais, Southeastern Brazil. Brazilian. Journal of Biology, 63(4): 635-646.

PREFASI, M. \& M. ABOAL. 1994. Estudio del Género Batrachospermum Roth: B. boryanum Sirod. y B. moniliforme Roth en la provincia de Castellón, España. Acta Botanica Malacitana, 19: 45-50.

ROS, M. D., E. LÓPEZ-JIMÉNEZ \& M. ABOAL. 1997. Primera cita de Hildenbrandia angolensis Welwitsch ex W. West \& G.S. West (Hildenbran- diales, Rhodophyceae), para la flora algal epicontinental española. Anales del Jardín Botánico de Madrid, 55(2): 458-460.

SABATER, S., M. ABOAL \& J. CAMBRA. 1989. Nuevas observaciones de rodofíceas en aguas epicontinentales del NE y SE de España. Limnetica, 5: 93-100.

SHEATH, R. G. \& J. M. BURKHOLDER. 1985. Characteristics of softwater streams in Rhode Island. II. Composition and seasonal dynamics of macroalgal communities. Hydrobiologia, 128: 109-118.

SHEATH, R. G. \& J. D. WEHR. 2003. Freshwater Algae of North America: Ecology and Classification. Academic Press. San Diego. CA. 918 pp.

SKUJA, V. H. 1926. Eine neue Süsswasserbangiacee Kyliniella latvica n.g., n. sp. Acta Horti Botanici Universitatis Latviensis, 1: 1-5.

VIS, M. L. \& R. G. SHEATH. 1993. Distribution and systematics of Chroodactylon and Kyliniella (Porphyridiales, Rhodophyta) from North American streams. Japanese Journal of Phycology, 41: 237-241.

YOON, H. S., K. M. MÜLLER, R. G. SHEATH, F. D. OTT \& D. BHATTACHARYA. 2006. Defining the major lineages of red algae (Rhodophyta). Journal of Phycology, 42: 482-492. 